\title{
Comentarios al editorial de Karol Kumpfer y Jeannette L. Johnson
}

\author{
Roberto Secades Villa; José Ramón Fernández Hermida
}

Grupo de Conductas Adictivas.

Departamento de Psicología. Universidad de Oviedo.

Enviar correspondencia a:

Roberto Secades Villa. Facultad de Psicoloía-Universidad de Oviedo. Plaza Feijoo s/n. 33003 Oviedo. Correo electrónico: secades@uniovi.es

$\mathbf{L}$ os factores de riesgo y protección identificados en los estudios españoles no difieren de los encontrados en las investigaciones realizadas en otros países, preferentemente anglosajones (Secades-Villa, Fernández-Hermida y Vallejo, 2004). Así, se ha demostrado que la función de socialización de la familia es un elemento esencial para la prevención del abuso de drogas de los adolescentes. Los déficit en la función de socialización se expresan mediante la pérdida de control sobre los hijos, la presencia de consumo de drogas y de actitudes tolerantes hacia las mismas y los conflictos familiares. La relación entre estos tres grandes factores de riesgo familiar y el consumo de drogas no es lineal, ni simplemente aditiva (Becoña, 2001). Estos tres factores no son independientes entre sí, y la simple presencia de uno de ellos, puede actuar como detonante que incremente la probabilidad de que surjan los otros dos. Además, la relación con el consumo de drogas aparece mediada por la presencia de otros factores de riesgo externos e incluso internos a la familia, alguno de carácter biológico, como la herencia. Sin embargo, la persistencia con la que estos grandes factores aparecen en la investigación, hace que sea lógica su inclusión como objetivos prioritarios de los programas de prevención.

Es en parte cierto que, contrariamente a lo que ha demostrado la investigación, ha existido cierta tendencia a no valorar la relación que existe entre la dinámica familiar y el riesgo de consumo en los hijos. Un tercio de los padres no cree que las malas relaciones familiares sean un factor de riesgo y la mayoría está a favor de que se reduzca el consumo mediante normas legales o a través de la formación escolar (Pinazo Hernandis y Pons Diez, 2001).

Pero también es cierto que a esta falsa creencia se debe añadir el hecho de que, al menos por lo que se refiere al caso de España, los padres han sido bombardeados sistemáticamente con mensajes que en muchas ocasiones van en el sentido contrario a las evidencias de los hallazgos empíricos.
Por una parte, es fácil de constatar la presencia de una actitud social indulgente con el uso de las drogas legales y hacia algunas ilegales, como el cannabis. Se trata de mensajes provenientes de diferentes fuentes (medios de comunicación, "intelectuales," mediadores sociales e, incluso, profesionales y expertos en drogas) que tienen como consecuencia una reducción de la percepción del riesgo asociado al consumo de estas sustancias. Esta posición es en ocasiones asumida por los padres y es perfectamente percibida por los hijos (Secades Villa y Fernández Hermida, 2001).

Por otra parte, es evidente que durante los últimos años en nuestro país, desde muchas instancias públicas y privadas se ha "adoctrinado" a los padres acerca de las bondades de lo que se llamaría un estilo educativo permisivo, basado en un déficit de control y en la ausencia de normas y castigos. Se podría afirmar que el estilo permisivo ("la autoridad compartida", según defendía recientemente una Consejera de Educación de una Comunidad Autónoma española) ha sido claramente el estilo que ha predominado en nuestra sociedad durante bastantes años. Este hecho se refleja, incluso, en las normas y leyes que rigen nuestro sistema educativo.

Obviamente, todas estas creencias trabajan en contra de los objetivos de la prevención y de la implicación de los padres en los programas de prevención y obligan a tomarlas en consideración si se quiere tener éxito con los mismos.

Sin embargo, parece existir muy poco apoyo y convicción en la prevención, y en particular, a la prevención familiar, por varios motivos. En primer lugar, los resultados de las campañas preventivas han de ser valorados a largo plazo y, con frecuencia, los responsables políticos que deben financiar estas campañas tienden a buscar resultados inmediatos, y esto no es posible. Esto es, sin duda, un inconveniente muy importante para la necesidad de la diseminación de los programas a la que se alude en la Editorial. En segundo lugar, en muchas ocasiones, la prevención que se 
hace no es la más adecuada, en términos de calidad técnica y científica, y por tanto, los resultados tampoco son positivos. Una prevención exitosa debe estar fundamentada en programas de eficacia contrastada, este es un asunto sobre el que se insiste en la Editorial y con el que estamos de acuerdo. Sin embargo, los gestores de la prevención (políticos y técnicos) no parecen inclinados a aceptar esa recomendación. Cada vez son más los programas de prevención que se ofertan, sin que haya una mínima garantía de calidad ni del programa ni de su aplicación. En la editorial se menciona que sólo un $10 \%$ de los programas de prevención familiar que se aplican (entendemos que en USA) tienen apoyo empírico. Este dato es sorprendente dado el esfuerzo que se hace en ese país para acreditar y difundir los buenos programas. ¿Cuántos programas de prevención, no sólo familiar, de los que se aplican en nuestro país han pasado por los requisitos que se exigen para alcanzar los niveles de Ejemplaridad (Exemplary) I y II que se mencionan en la editorial? ¿Es este un asunto de mera desidia o esta contumacia en el mal camino se debe a problemas más profundos de la prevención? Por último, ninguna estrategia de prevención opera en el vacío. Un programa de prevención familiar debe estar enmarcado en una estrategia preventiva que tenga en cuenta no sólo el marco escolar, sino también unas normas sociales y legislativas que den apoyo a los objetivos que se pretenden alcanzar. La actividad preventiva es una acción a largo plazo, de una gran complejidad técnica, y debe estar apoyada en una estructura profesional que le de apoyo y continuidad. Hoy por hoy esa estructura técnica no existe en España y ese es un grave handicap para alcanzar una efectividad apreciable.

Como bien afirma Karol Kumpfer, hoy en día se dispone de programas de prevención familiar eficaces, como los que se nombran en la Editorial. En la revisión Cochrane citada en el Editorial, se encuentra que el SFP tiene un NNT (Number Needed to Treat) de 9, o lo que es lo mismo, una efectividad en el $11 \%$ de los casos tratados a la hora de reducir las conductas peligrosas de consumo de alcohol (Foxcroft, Ireland, Lister-Sharp, Lowe y Breen, 2002). No está nada mal, pero hay que tener en cuenta que los datos están obtenidos de una investigación llevada a cabo por los defensores del programa, y es de suponer que realizada con toda la motivación y medios. ¿Qué sucederá en contextos de aplicación normalizados, no experimentales? Es un hecho que dichos programas distan mucho de estar exentos de graves problemas cuando se aplican en contextos reales. Así, el nivel de participación de los padres en los programas de prevención es extremadamente bajo. Las tasas de participación suelen estar por debajo del $20 \%$ o incluso del $5 \%$ (Al-Halabi, Secades-Villa, Errasti, Fernández-Hermida, Garcia-Rodríguez y Carballo, 2006). Por otra parte, en el momento que la actividad preventiva exige un esfuerzo continuado, se produce una alta tasa de abandono. Con esas condiciones no es extraño que "motivar" sea un verbo esencial cuando se conjuga con la palabra "prevención".

Puede resultar paradójica la discrepancia entre la altísima disponibilidad aparente de los padres a participar en los programas de prevención y las dificultades para el reclutamiento cuando se ponen en marcha. Es posible que las respuestas de los padres estén influidas por el estado de opinión de que la prevención familiar en el campo de las drogodependencias es muy importante, pero que sin embargo no se crean tal afirmación. Esta creencia explicaría su inicial disposición a participar, pero, también, su escasa inclinación a dedicar mucho esfuerzo. Se ha sugerido también que una posible razón para esta actitud se encuentre en la baja validez aparente de las estrategias preventivas, o si se quiere, de su baja aceptabilidad para sus destinatarios.

En todo caso, éste es un asunto fundamental que está aún por resolver y que probablemente requiera una gran cantidad de recursos e imaginación, para obtener resultados no siempre satisfactorios.

Por otra, las familias que asisten a estos programas tienen un perfil de bajo riesgo (bajo conflicto familiar, estilo educativo apropiado, actitudes negativas hacia el uso de drogas, lazos positivos entre padres e hijos, buena comunicación familiar y bajo consumo de drogas de los hijos), mientras que los padres con mayor riesgo evitan asistir. Esta evidencia nos conduce directamente hacia la necesidad de desarrollar de forma paralela a los programas de prevención universal, las intervenciones de carácter selectivo e indicado, dirigidas a grupos de riesgo y a jóvenes que, como se menciona en el Editorial, son los más efectivos, ya que concentran los escasos recursos en aquellos que más los necesitan. Pero, como ya se ha dicho anteriormente, una acción efectiva en este ámbito tiene que apoyarse en un soporte organizativo y técnico que hoy apenas existe. Mientras esta carencia no se satisfaga, la prevención familiar o de cualquier otro tipo no será algo más que un buen tema de investigación o un buen motivo para la propaganda.

\section{REFERENCIAS}

Al-Halabi Díaz, S., Secades Villa, R., Errasti Pérez, J.M., Fernández Hermida, J.R., Garcia Rodríguez, O. y Carballo Crespo, J.L. (2006). Family predictors of parent participation in adolescent drug abuse prevention program. Drug and Alcohol Review, 25, 323-327.

Becoña-Iglesias, E. (2001). Factores de riesgo y protección familiar para el uso de drogas. En R. Secades Villa y J. 
R. Fernández Hermida (Eds.), Intervención Familiar en la Prevención de las Drogodependencias. Madrid: Plan Nacional sobre la Drogas.

Foxcroft DR, Ireland D, Lister-Sharp DJ, Lowe G, Breen R. (2002) Prevención primaria para el abuso de alcohol en los jóvenes (Revisión Cochrane traducida). En: La Biblioteca Cochrane Plus, 2006 Número 4. Oxford: Update Software Ltd. Disponible en: http://www.update-software.com. (Traducida de The Cochrane Library, 2006 Issue 4. Chichester, UK: John Wiley \& Sons, Ltd.).

Pinazo Hernandis, S., y Pons Diez, J. (2001). Estudio empírico sobre la implicación de los padres en los programas preventivos del consumo de drogas. En R. Secades
Villa y J. R. Fernández Hermida (Eds.), Intervención Familiar en la Prevención de las Drogodependencias. Madrid: Plan Nacional sobre las Drogas.

Secades Villa, R., y Fernández Hermida, J. R. (2001). Factores de riesgo familiares para el uso de drogas: Un estudio empírico español. En R. Secades Villa y J. R. Fernández Hermida (Eds.), Intervención Familiar en la Prevención de las Drogodependencias. Madrid: Plan Nacional sobre las Drogas.

Secades Villa, R., Fernández Hermida, J.R. y Vallejo Seco, G. (2004). Family risk factors for adolescent drug misuse in Spain. Journal of Child and Adolescent Substance Abuse, 14, 3, 1-15. 
